\title{
Editorial
}

\section{Nanoparticle delivery of mitoprotective agents to target ischaemic heart disease}

Sang-Bing Ong ${ }^{1,2}$, Shengjie Lu², Khairunnisa Katwadi ${ }^{1,2}$, Nur Izzah Ismail ${ }^{1,4}$, Xiu-Yi Kwek ${ }^{1,2}$, Derek J Hausenloy ${ }^{1,2,3,5}$

${ }^{1}$ Cardiovascular and Metabolic Disorders Program, Duke-NUS Medical School, Singapore. ${ }^{2}$ National Heart Research Institute Singapore, National Heart Centre Singapore, Singapore, Singapore.

${ }^{3}$ The Hatter Cardiovascular Institute, Institute of Cardiovascular Science, University College London, London, UK.

${ }^{4}$ Department of Biomedical Engineering, Faculty of Engineering, University of Malaya, Malaysia.

${ }^{5}$ Yong Loo Lin School of Medicine, National University Singapore, Singapore

Keywords: Nanoparticles, mitoprotective agents, cardioprotection

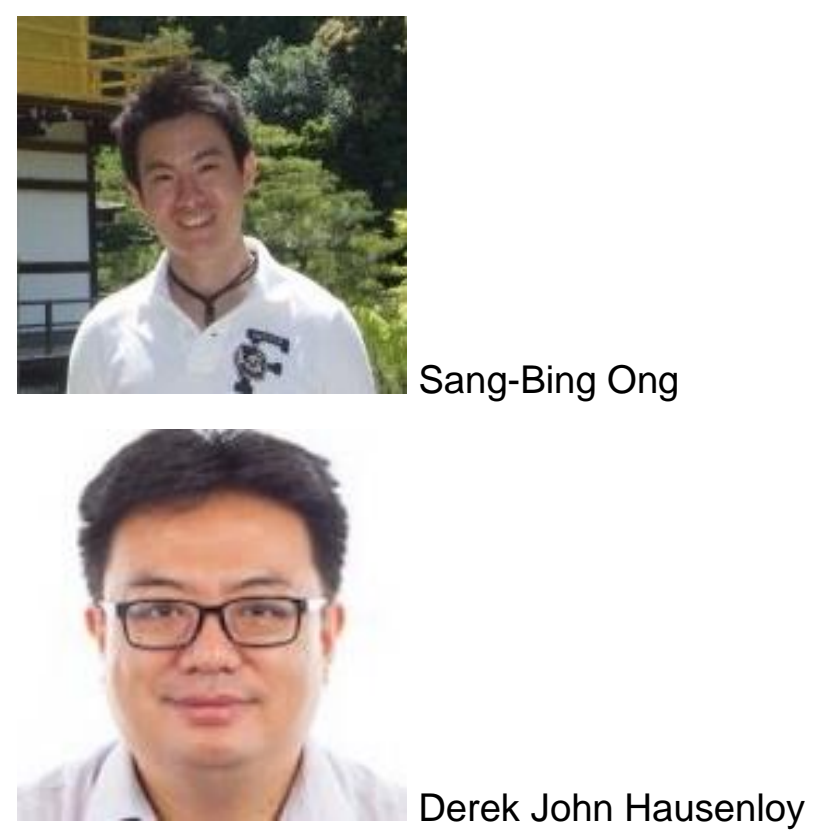

\section{Corresponding author:}

Dr Sang-Bing Ong

Cardiovascular \& Metabolic Diseases Program, Duke-National University of Singapore Medical School, 8 College Road, Singapore 169857.

Email: ongsangbing@duke-nus.edu.sg 


\section{ABSTRACT}

Mitochondrial dysfunction constitutes a critical determinant of myocardial reperfusion injury following acute myocardial infarction (AMI). Mitochondria are therefore important therapeutic targets for limiting myocardial infarct (MI) size and preventing heart failure in patients with ischaemic heart disease (IHD). Recently published clinical trials using mitoprotective agents targeting the mitochondrial permeability transition pore (mPTP) (cyclosporine-A (CsA) [1] or TRO40303 [2]) and mitochondrial cardiolipin (MTP-131) [3] have however, failed to demonstrate any reduction in $\mathrm{MI}$ size or improvement in clinical outcomes following AMI. This may have been, in part, due to the failure to deliver the agents to the mitochondria in sufficient concentrations. In this regard, research studies are investigating nanoparticles to improve the delivery of mitoprotective agents to cardiac mitochondria.

\section{Nanodelivery systems to target the mitochondria}

Nano-delivery systems have been designed to utilize the high negative mitochondrial membrane potential (approximately $-200 \mathrm{mV}$ ), and protein import machinery of mitochondria. In addition, the nano-carriers need to: (1) be non-cytotoxic with minimal side effects; (2) remain intact prior to reaching the target site; and (3) enhance the delivery and efficacy of the selected mitoprotective agent. However, the random interaction of drug molecules with mitochondria may not occur naturally, due to the inherent nature of the drug and the complex dual-membrane structure of mitochondria. Therefore the drug has to be designed specifically for mitochondrial localization or be transported into mitochondria by an appropriate mitochondria-targeted delivery system. Moreover, depending on the desired effects, the drugs should preferably, be able to target the different regions of the mitochondria, e.g. cristae, inner mitochondrial membrane or matrix. 
Nanodelivery of drugs to the cardiac mitochondria have mainly focused on the use of nanovesicles or nanoparticles. Nanovesicles targeting mitochondria include DQAsomes (DeQuAlinium-based liposome-like vesicles) and phospholipid vesicles with surface modification using triphenylphosphonium (TPP) cations (a concept applied to MitoQ - coenzyme-Q conjugated to TPP). Nanoparticles comprising of either solid (gold, zinc or silica), quantum dots or polymeric micelles can be targeted towards mitochondria using mitochondriotropic moieties such as rhodamine, methyl-TPP and dequalinium chloride.

Gold nanoparticles with diameter of $3 \mathrm{~nm}$ (3-nm AuNPs) have been demonstrated to enter mitochondria of permeabilised rat ventricular cardiomyocytes, and enter isolated cardiac mitochondria via the voltage-dependent anion channel (VDAC). Functionalisation of the AuNPs which serve to provide specific receptor sites and make the particle 'organic' have been performed through the attachment of mitochondriotropic ligands using phosphonioalkylthiosulfate zwitterions. These AuNPs have been used to protect against isoproterenol-induced myocardial injury [4], and as anti-inflammatory therapy for myocardial infarction [5].

The dual-function MITO-Porter (DF-MITO-Porter) - a nano device for mitochondrial delivery based on the concept of both high-density octaarginine (R8)-modified liposomes and the conventional MITO-Porter has also been described (in HeLa cells and rat liver homogenates) [6]. The nanoparticles carrying DNasel [7] or bongkrekic acid [8] were coated with a mitochondriafusogenic lipid envelope (inner), and endosome-fusogenic lipid envelope (outer). The R8 acts as a cytoplasmic-delivery device in the outer envelope and as a mitochondrial-targeting device in the inner envelope.

Another form of mitochondrial-targeting nanodelivery system are polymeric micelles comprising of an inner hydrophobic core which can encapsulate poorly water-soluble drugs and control their release, and a hydrophilic outer shell which provides aqueous solubility. Other 
systems include the mimetic of envelope-type viruses - the multifunctional envelope-type nanodevice (MEND) or water soluble fullerene derivatives that accumulate in mitochondria.

\section{Nanoparticle delivery of mitoprotective agents to cardiac mitochondria}

Several cardioprotection studies have used nanoparticles to target the heart [9-12], albeit not the mitochondria directly. The following sections will describe some of the nanoparticles that target the mitochondria directly. MitoQ® (MitoQ Ltd, Auckland, New Zealand) which is taken up by and enriched at the matrix surface of the inner mitochondrial membrane exhibits anti-oxidative properties. MitoQ comprises of an antioxidant coenzyme Q linked to the TPP cation, via a 10carbon alkyl chain. This compound has been administered safely long term to mice and is able to accumulate in the heart, brain, skeletal muscle, liver and kidney [13]. In the settings of acute IRI, $500 \mu \mathrm{M}$ MitoQ administered in drinking water for 2 weeks reduced Ml size in a Langendorffperfused heart in both mice [14] and rats [15].

CsA-encapsulated bioabsorbable poly-lactic/glycolic acid (PLGA) nanoparticles injected intravenously in mice at the onset of reperfusion was found to localize to mitochondria and protect against mPTP opening, reduce MI size and prevent left ventricular remodeling [16]. The tagged nanoparticles were distributed in the $\mathrm{Ml}$ area and the area-at-risk, even at 3 hours postreperfusion. The PLGA-nanoparticle mediated CsA delivery system had three beneficial effects: (1) The level of CsA in the nanoparticles could be maintained at a lower effective dose of $1.0 \mathrm{mg} / \mathrm{kg}$ thereby minimizing potential toxicity and off-target effects of $\mathrm{CsA}$; $(2)$ the local concentration of CsA in the ischaemic area of the myocardium was increased 5 -fold during the early phase (5 minutes) reperfusion reperfusion indicating improved delivery of CsA to the myocardium; and (3) the protective effect was 25 -fold greater when compared to CsA treatment alone [16]. The failure of the CIRCUS trial to demonstrate improved clinical outcomes with CsA 
administration at the time of reperfusion to AMI patients [1], may have in part be due to a failure to deliver CsA at sufficient concentrations to cardiac mitochondria, and this may be overcome using nanoparticle-mediated delivery of CsA.

Similarly, pre-treatment with PLGA nanoparticles containing mdivi-1, a small molecule inhibitor of the mitochondrial fission protein Drp1, with subsequent localization at the cytosol and mitochondria protected rat neonatal cardiomyocytes against $\mathrm{H}_{2} \mathrm{O}_{2}$-induced oxidative stress, compared to using mdivi-1 alone [17]. The localisation and beneficial effect were also corroborated in Langendorff-perfused mouse hearts delivered with PLGA-mdivi-1 through the coronary arteries at the time of reperfusion, via a cyclophilin $D(C y p D)$-independent but Baxdependent mechanism [17].

More recently, autologous mitochondria have been perfused directly into the coronary arteries at the onset of reperfusion in rabbit hearts subjected to 30 minutes of regional ischaemia and reperfused for 120 minutes - MI size was significantly reduced while post-ischaemic myocardial function was enhanced [18]. Although the authors initially tagged the mitochondria with 18Frhodamine $6 \mathrm{G}$ and $30 \mathrm{~nm}$ iron oxide nanoparticles, these labelled mitochondria were solely used for detection of localisation sites following direct injection into the ischaemic region or delivery by vascular perfusion through the coronary arteries at the onset of reperfusion in Langendorffperfused rabbit hearts subjected to 30 minutes of global ischaemia and reperfusion for 10 minutes [18]. Whether encapsulation of mitochondria in nanoparticles will further enhance the efficacy/benefits of direct intracoronary mitochondria delivery remains to be investigated.

\section{Clinical Implementation}

MitoQ has been developed by Antipodean Pharmaceuticals Inc. as a pharmaceutical using the methanesulfonate counter-anion and complexing it with $\beta$-cyclodextrin for a stable formulation. 
MitoQ has been investigated in a clinical Phase I trial, and has exhibited good pharmacokinetic behavior with $10 \%$ oral bioavailability and is currently in clinical trials for Parkinson's disease and chronic hepatitis $\mathrm{C}$. Whether it can be used to target $\mathrm{AMI}$ in the clinical setting needs to be investigated.

Although yet to be used as specific mitochondrial-drug carriers in clinical trials, PLGA polymers as drug carriers have received biosafety approval for human use by the US Food and Drug Administration, the European Medicine Agency, and the Japanese regulatory agency (PMDA). It is important to note that there have been reports of detrimental effects of certain nanoparticles as vectors such as silica [19] and zinc oxide [20] nanoparticles. Further investigations are warranted to determine whether these effects are restricted to these particular particles or in the specific settings of the reported studies.

\section{Conclusion \& future perspective}

Mitochondria are attractive organelles for drug targeting in patients with IHD, given that mitochondria govern cell fate following acute IRI. Nonetheless, delivery of these drugs to mitochondria requires the passage through the plasma and mitochondrial membranes. In this regard, nanoparticle-based carriers may help overcome this hurdle of transporting drugs to mitochondria. However, further investigation is needed to evaluate the safety and long-term efficacy of these nanoparticles, particularly in humans. Further advancements should also be focused on designing organ-specific mitochondrial-targeting particles since the cargo should accumulate preferentially both at the desired target tissue and site of action.

\section{Funding}


Sang-Bing Ong is supported by the Singapore Ministry of Health's National Medical Research Council under its Open Fund-Young Individual Research Grant (OF-YIRG) NMRC/OFYIRG/0021/2016 as well as a Khoo Postdoctoral Fellowship Award (KPFA) - DukeNUS-KPFA/2016/0010 from the Estate of Tan Sri Khoo Teck Puat, Singapore. Derek Hausenloy is supported by the British Heart Foundation (FS/10/039/28270), the Rosetrees Trust, Duke-NUS Medical School, and the National Institute for Health Research University College London Hospitals Biomedical Research Centre.

\section{Conflict of Interest}

All authors declare no conflict of interest.

References

1. Cung T-T, Morel O, Cayla G, et al. Cyclosporine before PCl in Patients with Acute Myocardial Infarction. N. Engl. J. Med. [Internet]. 373(11), 1021-1031 (2015). Available from: http://www.ncbi.nlm.nih.gov/pubmed/26321103.

2. Atar D, Arheden H, Berdeaux A, et al. Effect of intravenous TRO40303 as an adjunct to primary percutaneous coronary intervention for acute ST-elevation myocardial infarction: MITOCARE study results. Eur. Heart J. [Internet]. 36(2), 112-9 (2015). Available from: https://academic.oup.com/eurheartj/article-lookup/doi/10.1093/eurheartj/ehu331.

3. Gibson CM, Giugliano RP, Kloner RA, et al. EMBRACE STEMI study: a Phase 2a trial to evaluate the safety, tolerability, and efficacy of intravenous MTP-131 on reperfusion injury in patients undergoing primary percutaneous coronary intervention. Eur. Heart $\mathrm{J}$. [Internet]. 37(16), 1296.1-1303 (2016). Available from: http://www.ncbi.nlm.nih.gov/pubmed/26586786.

4. Vinodhini A, Govindaraju K, Singaravelu G, Mohamed Sadiq A, Kumar VG. Cardioprotective potential of biobased gold nanoparticles. Colloids Surfaces $B$ Biointerfaces. 117, 480-486 (2014).

5. Somasuntharam I, Yehl K, Carroll SL, et al. Knockdown of TNF- $\alpha$ by DNAzyme gold nanoparticles as an anti-inflammatory therapy for myocardial infarction. Biomaterials 
[Internet]. 83, 12-22 (2016). Available from:

http://www.ncbi.nlm.nih.gov/pubmed/26773660.

6. Yamada Y, Furukawa R, Yasuzaki Y, Harashima H. Dual Function MITO-Porter, a Nano Carrier Integrating Both Efficient Cytoplasmic Delivery and Mitochondrial Macromolecule Delivery. Mol. Ther. [Internet]. 19(8), 1449-1456 (2011). Available from:

http://www.ncbi.nlm.nih.gov/pubmed/21694702.

7. Yamada $\mathrm{Y}$, Harashima $\mathrm{H}$. Delivery of bioactive molecules to the mitochondrial genome using a membrane-fusing, liposome-based carrier, DF-MITO-Porter. Biomaterials [Internet]. 33(5), 1589-1595 (2012). Available from:

http://www.ncbi.nlm.nih.gov/pubmed/22105068.

8. Yamada Y. [Development of the MITO-porter, a nano device for mitochondrial drug delivery via membrane fusion]. Yakugaku Zasshi [Internet]. 134(11), 1143-55 (2014). Available from: http://www.ncbi.nlm.nih.gov/pubmed/25366911.

9. Bae S, Park M, Kang C, et al. Hydrogen Peroxide-Responsive Nanoparticle Reduces Myocardial Ischaemia/Reperfusion Injury. J. Am. Heart Assoc. [Internet]. 5(11), e003697 (2016). Available from: http://www.ncbi.nlm.nih.gov/pubmed/27930351.

10. Dvir T, Bauer M, Schroeder A, et al. Nanoparticles Targeting the Infarcted Heart. Nano Lett. [Internet]. 11(10), 4411-4414 (2011). Available from:

http://www.ncbi.nlm.nih.gov/pubmed/21899318.

11. Margulis K, Neofytou EA, Beygui RE, Zare RN. Celecoxib Nanoparticles for Therapeutic Angiogenesis. ACS Nano [Internet]. 9(9), 9416-9426 (2015). Available from:

http://www.ncbi.nlm.nih.gov/pubmed/26244654.

12. Alejandro R-LM, Pablo M-CP, Hector R-H, et al. Evaluation of vascular tone and cardiac contractility in response to silver nanoparticles, using Langendorff rat heart preparation. Nanomedicine Nanotechnology, Biol. Med. [Internet]. (2017). Available from: http://www.ncbi.nlm.nih.gov/pubmed/28214609.

13. Smith RAJ, Porteous CM, Gane AM, Murphy MP. Delivery of bioactive molecules to mitochondria in vivo. Proc. Natl. Acad. Sci. [Internet]. 100(9), 5407-5412 (2003). Available from: http://www.ncbi.nlm.nih.gov/pubmed/12697897.

14. Neuzil J, Widén C, Gellert N, et al. Mitochondria transmit apoptosis signalling in cardiomyocyte-like cells and isolated hearts exposed to experimental ischaemiareperfusion injury. Redox Rep. [Internet]. 12(3), 148-162 (2007). Available from: http://www.ncbi.nlm.nih.gov/pubmed/17623522.

15. Adlam VJ, Harrison JC, Porteous CM, et al. Targeting an antioxidant to mitochondria decreases cardiac ischaemia-reperfusion injury. FASEB J. 19(9), 1088-95 (2005).

16. Ikeda G, Matoba T, Nakano Y, et al. Nanoparticle-Mediated Targeting of Cyclosporine A Enhances Cardioprotection Against Ischaemia-Reperfusion Injury Through Inhibition of Mitochondrial Permeability Transition Pore Opening. Sci. Rep. [Internet]. 6, 20467 (2016). Available from: http://www.ncbi.nlm.nih.gov/pubmed/26861678. 
17. Ishikita A, Matoba T, Ikeda G, et al. Nanoparticle-Mediated Delivery of Mitochondrial Division Inhibitor 1 to the Myocardium Protects the Heart From Ischaemia-Reperfusion Injury Through Inhibition of Mitochondria Outer Membrane Permeabilization: A New Therapeutic Modality for Acute Myocardial . J Am Hear. Assoc. 5(7), e003872 (2016).

18. Cowan DB, Yao R, Akurathi V, et al. Intracoronary Delivery of Mitochondria to the Ischaemic Heart for Cardioprotection. PLoS One [Internet]. 11(8), e0160889 (2016). Available from: http://www.ncbi.nlm.nih.gov/pubmed/27500955.

19. Guerrero-Beltrán CE, Bernal-Ramírez J, Lozano O, et al. Silica nanoparticles induce cardiotoxicity interfering with energetic status and $\mathrm{Ca2}+$ handling in adult rat cardiomyocytes. Am. J. Physiol. - Hear. Circ. Physiol. [Internet]. , ajpheart.00564.2016 (2017). Available from: http://www.ncbi.nlm.nih.gov/pubmed/28130337.

20. Zhao X, Ren X, Zhu R, Luo Z, Ren B. Zinc oxide nanoparticles induce oxidative DNA damage and ROS-triggered mitochondria-mediated apoptosis in zebrafish embryos. Aquat. Toxicol. [Internet]. 180, 56-70 (2016). Available from: http://www.ncbi.nlm.nih.gov/pubmed/27658222. 